
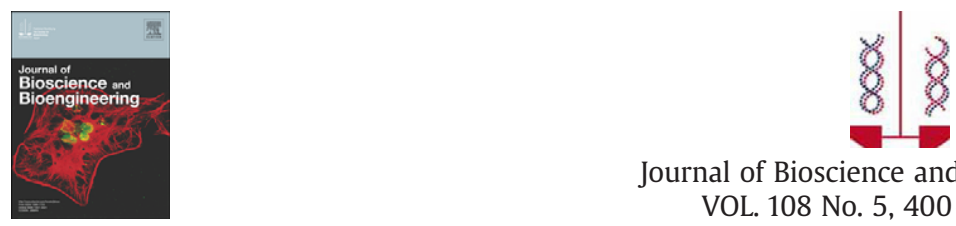

Journal of Bioscience and Bioengineering

VOL. 108 No. 5, $400-407,2009$

www.elsevier.com/locate/jbiosc

\title{
Comparison of archaeal and bacterial community structures in heavily oil-contaminated and pristine soils
}

\author{
Ruyin Liu, Yu Zhang, Ran Ding, Dong Li, Yingxin Gao, and Min Yang* \\ State Key Laboratory of Environmental Aquatic Chemistry, Research Center for Eco-Environmental Sciences, \\ Chinese Academy of Sciences, 100085, Beijing, China \\ Received 17 November 2008; accepted 16 May 2009
}

\begin{abstract}
Archaeal and bacterial community structures in heavily oil-contaminated and pristine soils were compared using denaturing gradient gel electrophoresis and 16S rRNA gene libraries. The results showed that archaeal diversity was more complex in the contaminated soil than in the uncontaminated control soil. Archaeal populations in the contaminated soil consisted mainly of Euryarchaeota, with abundant methanogen-like operational taxonomic units (OTUs) and OTUs related to the phylogenetically diverse group, candidate division I, corresponding to rice cluster V. In contrast, only halophilic archaea were found in the pristine soil. Bacterial community structures also differed significantly between the contaminated and pristine soils. More clones from the contaminated soil were related to known hydrocarbon-degrading bacteria, implying that microorganisms with the potential to degrade petroleum were well-established. These results provide further insights into the composition of microbial communities in oil-contaminated soils.
\end{abstract}

(c) 2009, The Society for Biotechnology, Japan. All rights reserved.

[Key words: Archaeal diversity; Bacterial diversity; Oil-contaminated soil; 16S rRNA gene; Denaturing gradient gel electrophoresis]

The toxic, mutagenic, and carcinogenic properties of petroleum mean that soil contamination by crude oil is an important environmental problem during oil drilling and production (1). Bacteria are important for the biodegradation of petroleum hydrocarbons, and many hydrocarbon-degrading bacteria have been isolated from different environments. These isolated bacteria are able to degrade alkanes and/or aromatic hydrocarbons under aerobic or anaerobic conditions. The bacteria have been assigned to a number of genera, including Pseudomonas, Alcanivorax, and Marinobacter etc. Some of them have been widely used for bioremediation of oil-contaminated environments $(2,3)$. Consequently, much research has focused on the characteristics of bacterial community structures in oil-contaminated sites, as well as changes in these community structures associated with oil contamination $(4,5)$.

Recent molecular-based studies have shown archaea, which were once thought to be synonymous with extreme environments, to be present in almost all the environments examined to date (6). Archaea have been detected in several oil-containing environments, such as petroleum reservoirs (7), underground crude oil storage cavities (8), and hydrocarbon-polluted aquifers (9). Furthermore, researchers have shown that archaea are involved in the mineralization of petroleum hydrocarbons, especially by methanogenesis under anaerobic conditions $(10,11)$. However, relatively little is known about archaeal community structures in heavily oil-contaminated soils.

\footnotetext{
* Corresponding author. Tel.: +8610 62923475; fax: +86 1062923541

E-mail address: yangmin@rcees.ac.cn (M. Yang).
}

Denaturing gradient gel electrophoresis (DGGE) fingerprinting and cloning and sequencing of polymerase chain reaction (PCR)amplified 16S rRNA gene fragments have been successfully used to analyze bacterial and archaeal community structures in a wide range of environmental samples $(12,13)$. PCR-DGGE has the advantage of quickly and economically analyzing the diversity of microbial assemblages present in different environments, and sequence analysis of clones from 16S rRNA gene clone libraries can provide detailed, reliable information about the structure and diversity of microbial communities in specific ecosystems. Thus, in the present study, DGGE and 16S rRNA gene libraries were used for the comparative analysis of the archaeal and bacterial communities in oil-contaminated and pristine soils.

\section{MATERIALS AND METHODS}

Soil samples The sampling sites, including oil-contaminated and pristine soils, were located in the Jidong Oilfield near Bohai Bay, China. The oil-contaminated site had been used as a storage area for petroleum leaks during petroleum exploitation for about 10 years. Oil stains were visible on the surface of the soil. The pristine site was located about $50 \mathrm{~m}$ from the oil-contaminated site. In December 2006, soil samples were collected from the top of each site $(0-2 \mathrm{~cm})$ using an ethanol-swabbed hand shovel Immediately after collection, samples were stored in sterilized glass jars at $4{ }^{\circ} \mathrm{C}$ Samples were collected from multiple areas within each site and mixed thoroughly to produce homogenized samples. Total petroleum hydrocarbon (TPH) concentration was determined by the method previously described (14). The saturated and aromatic components were analyzed by gas chromatography-mass spectrometry (Agilent 6890 / 5973, Santa Clara, CA, USA) (15). The total nitrogen (T-N) and total phosphorus (T-P) concentrations were determined as described previously (16). The physicochemical characteristics of the soil samples are shown in Table 1. 
DNA extraction The soil samples was washed for three times using phosphatebuffered saline (PBS, pH7.0) and centrifuged at $4{ }^{\circ} \mathrm{C}, 10,000 \mathrm{rpm}$ for $15 \mathrm{~min}$. Then the genomic DNA was extracted by the method described by Tsai et al (17).

PCR-DGGE In order to minimize PCR bias in PCR-DGGE and subsequent cloning steps, three separate reactions were run for each sample and the results were pooled. For bacteria, 16S rRNA gene fragments were amplified by PCR with primers 341F, using a 40-bp GC-clamp attached to the 5' end, and 534R (18). For archaea, 16S rRNA gene fragments were amplified using a nested PCR approach. The first-round PCR was performed with primers Arch571F and UA1406R (19), followed by amplification using primers Arch915F (20) with a GC-clamp, and UA1406R. The resulting amplification products were separated using DGGE with the Dcode Universal Mutation System (BioRad, Hercules, CA, USA). The PCR products $(20-25 \mu \mathrm{l})$ were loaded onto $6 \%(\mathrm{w} / \mathrm{v})$ polyacrylamide gels in Tris-acetate-EDTA buffer ( $\mathrm{pH}$ 7.4). The gels had denaturing gradients of $35-57 \%$ ( $100 \%$ denaturant contains $7 \mathrm{M}$ urea and $40 \%$ formamide) and were run at $150 \mathrm{~V}$ at $60{ }^{\circ} \mathrm{C}$ for $4.5 \mathrm{~h}$. After electrophoresis, the gels were stained for $15 \mathrm{~min}$ with ethidium bromide and photographed on a UV transillumination table using a Gel Documentation System (Bio-Rad Laboratories-Segrate, Milan, Italy). DGGE gels were processed using Bio-Rad Quantity One 4.3.0 software.

Cloning and sequencing of 16S rRNA gene The 16S rRNA gene fragments were amplified by PCR using primers Arch571F and UA1406R for archaea, and 27F and 1492R (21) for bacteria. PCR products were purified using TaKaRa purification kits. The amplified 16S rRNA gene fragments were cloned into the TOPO TA cloning vector pCR2.1, and then TOP10 chemically competent E. coli was transformed with the plasmids according to the manufacture's instructions (Invitrogen). Transformants were selected by blue-white selection on Luria-Bertani (LB) agar plates containing ampicillin $(100 \mu \mathrm{g} / \mathrm{ml})$. Cloned inserts were amplified from lysed colonies by PCR with plasmidvector specific primers M13F and M13R. Clones were sequenced with an ABI 3730 automated sequencer (Invitrogen, Shanghai, China).

Phylogenetic analysis The archaeal sequences sharing $97 \%$ or greater similarity were considered to represent the same operational taxonomic unit (OTU) using software DOTUR (http://schloss.micro.umass.edu/software/). Sequences were searched against the Ribosomal Database Project II (RDP II) release 9.49 and the GenBank database by using the BLASTn program to determine the nearest matches. Phylogenetic trees were constructed using MEGA, version 3.1 by the neighbor-joining algorithm and the Jukes-Cantor distance estimation method with bootstrap analyses for 1000 replicates was performed. The presence of chimera in the obtained sequences was determined by using the CHECK_CHIMERA (RDP; http://rdp.cme.msu.edu) prior to phylogenetic analysis.

Soil-slurry experiments The basal salt medium used in this study was composed of $5 \mathrm{mM} \mathrm{KH}_{2} \mathrm{PO}_{4}, 5 \mathrm{mM} \mathrm{K}_{2} \mathrm{HPO}_{4}$, and $14 \mathrm{mM} \mathrm{NH}_{4} \mathrm{Cl}$. Approximately $2 \mathrm{~g}$ of soil and $100 \mathrm{ml}$ of the sterile salt medium were dispensed into flasks $(300 \mathrm{ml}$ in capacity). Sterilized petroleum was added to each flask to a final amount of $120 \mathrm{mg} \mathrm{TPH}$. Flasks were incubated at $25^{\circ} \mathrm{C}$ with shaking at $150 \mathrm{rpm}$. Sterile controls were prepared under the same conditions by autoclaving the flasks for $20 \mathrm{~min}$ at $121^{\circ} \mathrm{C}$ before adding petroleum. All the slurry systems were prepared in triplicate. TPH was determined as described above.

Nucleotide sequence accession numbers The partial $16 \mathrm{~S}$ rRNA gene sequences that were determined have been deposited in the GenBank, nucleotide sequence databases under accession nos. EU735565-EU735702.

\section{RESULTS}

DGGE analysis PCR products from DNA extracted from the pristine and oil-contaminated soils showed the expected 491-bp archaeal PCR fragment and the 193-bp bacterial fragment. The archaeal populations in the two samples were clearly different (Fig. 1A). Archaeal diversity in the pristine soil was low, with only one intense band visible. More DGGE bands were produced from archaea found in the contaminated soil. There was only $35.5 \%$ similarity in the archaeal DGGE profiles between the contaminated and pristine soils (Dice's coefficient) (Fig. 1A). The bacteria from the two samples,

TABLE 1. Characteristics of soil samples used in this study.

\begin{tabular}{lcc}
\hline Parameter & Pristine soil & Contaminated soil \\
\hline TPH (mg/kg) & $<100$ & 52709 \\
Petroleum component (\%) & 0 & 54.8 \\
Saturates & 0 & 13.6 \\
Aromatics & 0.087 & 0.076 \\
Total N (\%) & 0.046 & 0.041 \\
Total P (\%) & 5.5 & 4.2 \\
Salt content (g/Kg) & 12 & 16 \\
Moisture (\%) & 8.7 & 8.6 \\
pH & &
\end{tabular}

Values are means of triplicates.
A

1

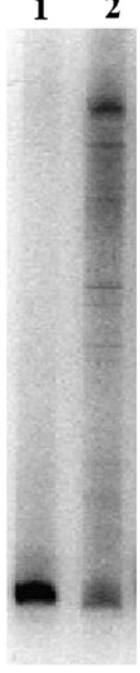

B

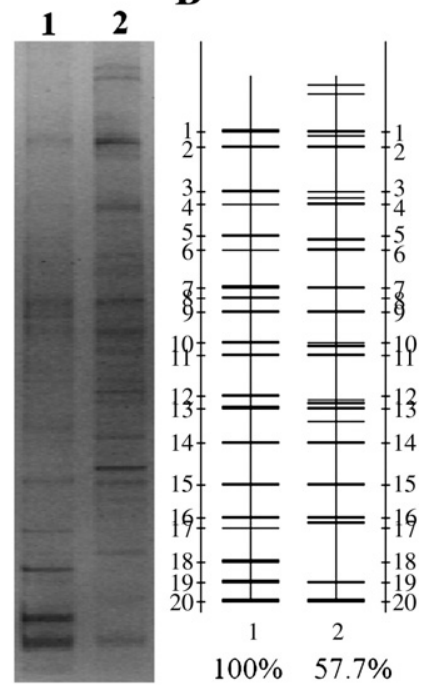

FIG. 1. Denaturing gradient gel electrophoresis profiles for partial 16S rDNA fragments showing archaeal (A) and bacterial (B) diversities in the pristine (lane 1) and oilcontaminated (lane 2) soil samples. The diagrams were generated using Quantity One 4.3.0 software.

however, showed more diversity (Fig. 1B), and the DGGE patterns for bacteria from the two soil samples showed a similarity of $57.7 \%$.

Diversity of $16 \mathrm{~S}$ rRNA gene sequences Due to the fact that the number of bands generated by DGGE may not accurately reflect the number of different sequences present in a given DNA mixture (22), clone libraries were constructed to obtain more detailed information. Partial 16S rRNA gene fragments were PCR-amplified from the extracted DNA using the bacterial and archaeal specific primer sets, and four clone libraries were produced (SCA, archaea in the contaminated soil; SNA, archaea in the pristine soil; SC, bacteria in the contaminated soil; SN, bacteria in the pristine soil). For the two archaeal libraries, a total of 59 and 28 archaeal clones of around 840 nucleotides were obtained from the contaminated and pristine soils, respectively, and these were classified into 26 OTUs (SCA) and 10 OTUs (SNA) with a sequence similarity of 0.97 or greater, using DOTUR software. For each of the two bacterial libraries, 50 (SC) and 56 bacterial clones $(\mathrm{SN})$ were randomly selected. Four sequences from the contaminated soil were identified as chimeric and were excluded from further analyses.

All of the 10 archaeal OTUs derived from the pristine soil were affiliated with Halobacteriales (Fig. 2). Twenty-two OTUs from the contaminated soil (83\% of clones) fell into the Euryarchaeota (Fig. 2). Among these, seven OTUs (25.4\% of clones) were affiliated with known euryarchaeotic orders (three with Methanosarcinales, two with Methanomicrobiales and two with Halobacteriales); one OTU (SCA110) formed a monophyletic clade; the other 14 OTUs were not affiliated with any of the known orders and were divided into two order-level phylogenetic groups (candidate divisions I and II). The remaining four OTUs were grouped into Crenarchaeota, and were related to unidentified environmental clones (Table 2).

For the bacterial libraries, taxa that were common to both soils included species from Alpha-, Delta-, and Gammaproteobacteria, and Bacteroidetes (Table 3). Gammaproteobacteria predominated in both bacterial libraries (Fig. 3). The contaminated soil contained a smaller proportion of Gammaproteobacteria (32.6\% of clones) and Bacteroidetes clones (13\% of clones) than the pristine soil, but more Alpha- and Deltaproteobacteria clones (Table 3). Taxa that were unique to the contaminated soil included four phyla that were represented by three clones from the Planctomycetes, two clones of Chloroflexi, one clone of 


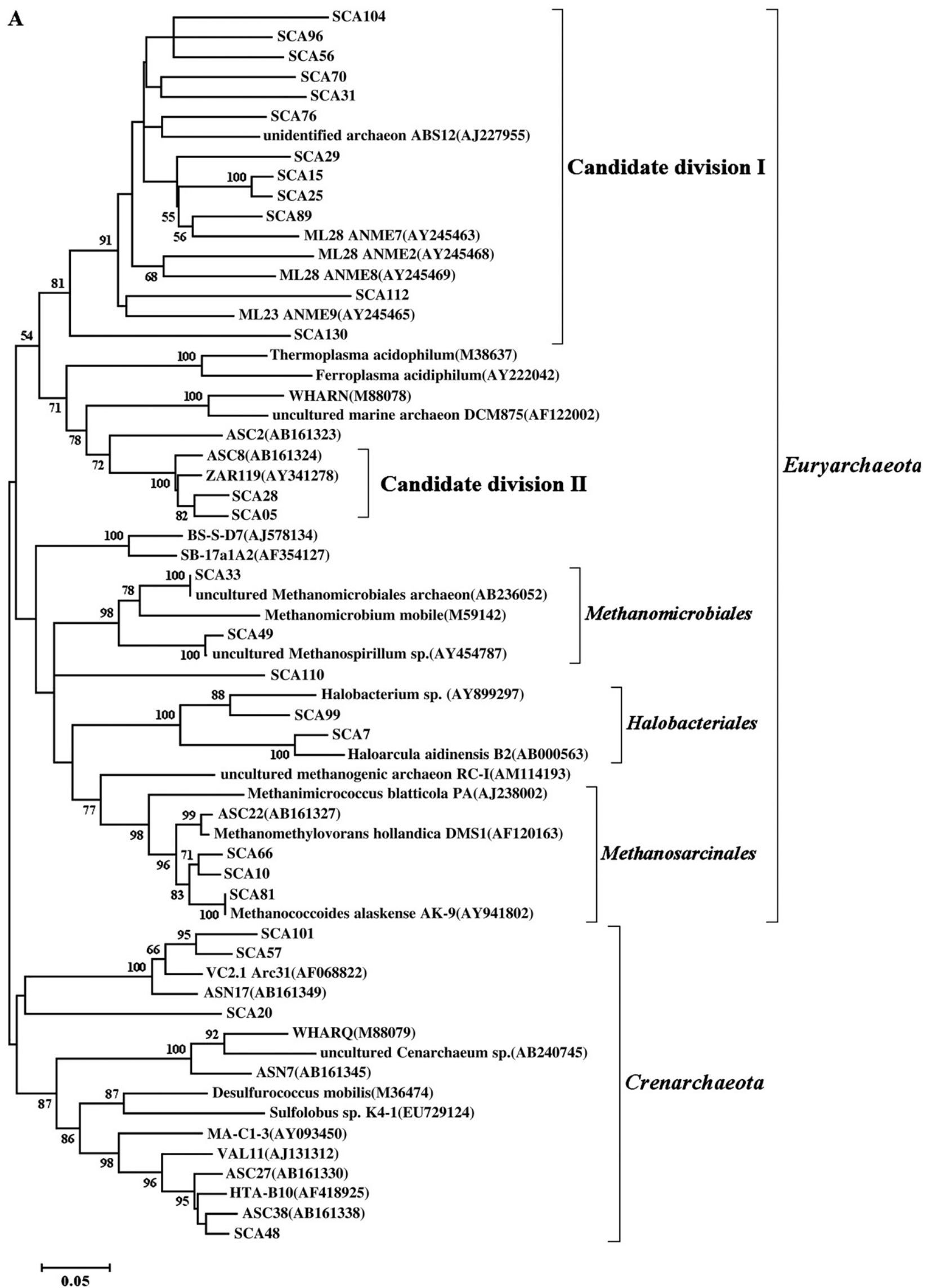




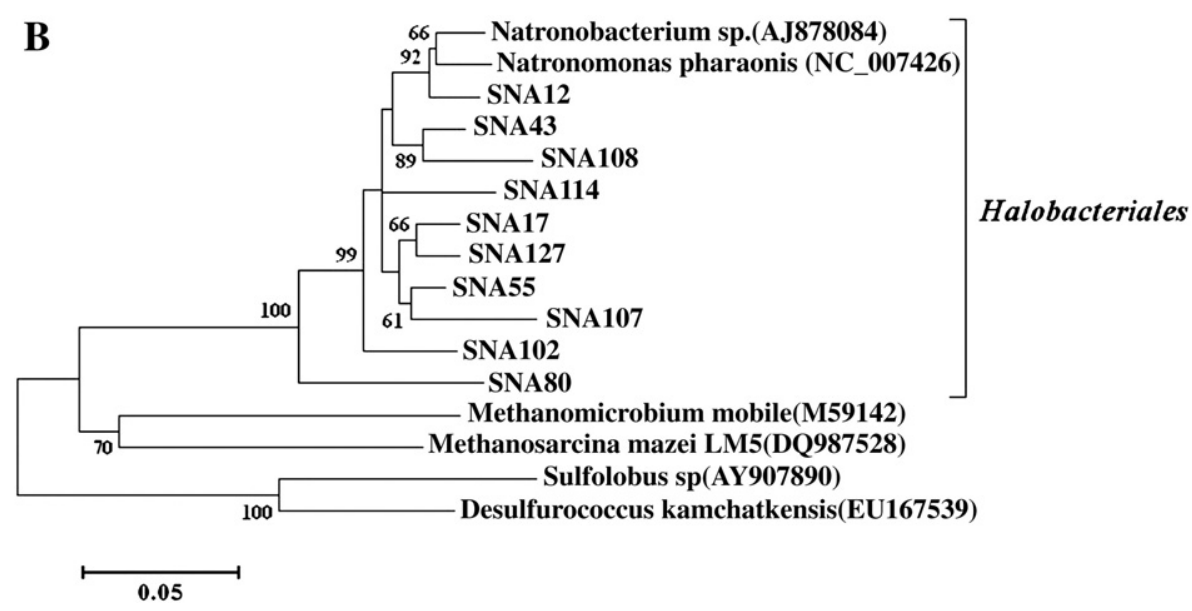

FIG. 2 (continued).

Acidobacteria, and two clones of Firmicutes. In contrast, Actinobacteria and Betaproteobacteria (each with five clones) were only detected in the pristine soil. In addition, three (SN) and four (SC) clones could not be classified into any known phyla.

Hydrocarbon degradation potential in the soil samples The potential of the contaminated and pristine soil samples for petroleum hydrocarbon degradation were compared in soil-slurry systems, and the results are shown in Fig. 4. The TPH removal rate by each soil was estimated by subtracting the average abiotic loss of TPH in three sterile controls from the loss in each sample flask. The system inoculated with the contaminated soil exhibited a much higher TPH removal rate than the system inoculated with the pristine soil. The 8day TPH removals were $87.6 \%$ and $45.8 \%$ for the contaminated and pristine soil-slurry systems, respectively, showing that the contaminated soil possessed a significantly higher TPH removal potential.

\section{DISCUSSION}

In this study, both DGGE profiles and analysis of archaeal libraries showed that archaeal community structure was more complex in heavily oil-contaminated soil than in pristine soil. All archaeal clones retrieved from the pristine soil were grouped into the order Halobacteriales, and were closely related to Natronomonas and Natronobacterium. A similar result was observed by Valenzuela-Encinas et al. in a study of analyzing the archaeal community in an alkaline-saline soil (23). In contrast, the archaeal community in the contaminated soil was more diverse. In addition to the Halobacteriales phylotypes (two OTUs associated with the genera Halobacterium and Haloarcula), phylotypes belonging to other order-level phylogenetic groups in the Euryarchaeota (Methanosarcinales, Methanomicrobiales, candidate divisions I and II), and Crenarchaeota were detected.

TABLE 2. Distribution of archaeal operational taxonomic units and related clone numbers in phylogenetic groups.

\begin{tabular}{|c|c|c|c|c|}
\hline \multicolumn{2}{|c|}{ Phylogenetic group } & \multicolumn{2}{|c|}{ Number of OTUs from } & \multirow{2}{*}{$\begin{array}{l}\text { Relative no. of clones } \\
\text { from contaminated } \\
\text { soil }\end{array}$} \\
\hline & & $\begin{array}{c}\text { Pristine } \\
\text { soil }\end{array}$ & $\begin{array}{c}\text { Contaminated } \\
\text { soil }\end{array}$ & \\
\hline \multirow[t]{5}{*}{ Euryarchaeota } & Candidate division I & - & $12(25)$ & $42.4 \%$ \\
\hline & Methanosarcinales & - & $3(5)$ & $8.5 \%$ \\
\hline & Methanomicrobiales & - & $2(8)$ & $13.6 \%$ \\
\hline & Halobacteriales & $10(28)$ & $2(2)$ & $3.4 \%$ \\
\hline & Candidate division II & - & $2(7)$ & $11.9 \%$ \\
\hline Crenarchaeota & Unknown & - & $4(10)$ & $16.9 \%$ \\
\hline
\end{tabular}

In the contaminated soil, five OTUs representing $22 \%$ of sequences belonged to methanogens. Three OTUs were closely affiliated with isolated Methanosarcinales species known to use methanol, methylamine, and related methyl-substrates for the production of methane (SCA10 with halophilic Methanohalophilus portucalensis, SCA66 with Methanolobus psychrophilus and SCA81 with Methanococcoides alaskense AK-9). Two OTUs (SCA49 and SCA33) were affiliated with isolated Methanomicrobiales species known to utilize $\mathrm{H}_{2}-\mathrm{CO}_{2}$ and formate: OTU SCA49 was closely related to mesophilic Methanospirillum hungatei; and OTU SCA33 was related to Methanoculleus receptaculi isolated from the Shengli oil field, China (95\% similarity). Previous studies have shown that methanogens were present in various oil-contaminated environments. Yoshida et al. detected clones related to Methanosaeta and Methanoculleus in crude-oil sludge (8). Kasai et al. found Methanosaeta and Methanomethylovorans belonging to the order Methanosarcinales in petroleum-contaminated unsaturated soil, but no methanogens in uncontaminated soil (24). Watanabe et al. demonstrated that archaea related to Methanosaeta and Methanomethylovorans, as well as Methanomicrobiales, were present in oil-contaminated groundwater (25). Most of these studies detected acetoclastic Methanosaeta. Acetoclastic methanogenesis has been suggested to be the major terminal electron-accepting process associated with anaerobic hydrocarbon degradation (9). However, acetoclastic Methanosaeta was not detected in the contaminated soil in the present study, and all of the OTUs belonging to Methanosarcinales were related to halophilic and methylphilic genera in the family Methanosarcinaceae. This may be related to the relatively high salt concentration in the contaminated soil (Table 1), and methyl compounds are thought to be the primary substrates for methanogens

TABLE 3. Distribution of bacterial clones in phylogenetic groups.

\begin{tabular}{|c|c|c|c|c|}
\hline \multirow{2}{*}{$\begin{array}{l}\text { Phylogenetic } \\
\text { group }\end{array}$} & \multicolumn{2}{|c|}{ Number of clones from } & \multicolumn{2}{|c|}{ Relative number of clones from } \\
\hline & Pristine soil & Contaminated soil & Pristine soil & Contaminated soil \\
\hline$\alpha$-Proteobacteria & 3 & 8 & $5.4 \%$ & $17.4 \%$ \\
\hline$\beta$-Proteobacteria & 5 & - & $8.9 \%$ & - \\
\hline$\gamma$-Proteobacteria & 28 & 15 & $50.0 \%$ & $32.6 \%$ \\
\hline$\delta$-Proteobacteria & 2 & 5 & $3.6 \%$ & $11.0 \%$ \\
\hline Bacteroidetes & 10 & 6 & $17.9 \%$ & $13.0 \%$ \\
\hline Planctomycetes & - & 3 & - & $6.5 \%$ \\
\hline Chloroflexi & - & 2 & - & $4.3 \%$ \\
\hline Acidobacteria & - & 1 & - & $2.2 \%$ \\
\hline Firmicutes & - & 2 & - & $4.3 \%$ \\
\hline Actinobacteria & 5 & - & $8.9 \%$ & - \\
\hline Unknown & 3 & 4 & $5.4 \%$ & $8.7 \%$ \\
\hline
\end{tabular}


A
${ }^{99}$ S1 $\mathrm{SC10}$ Marinobacter sp. MARC4V(DQ768635)

92 SC34

92 SC43

99 Marinobacter hydrocarbonoclasticus(DQ768638)

$62 \quad 5 C 108$

ASs2020(DQ665807)

-SC152

99 SC13

99 - Halomonas salina(AY505525)

91 SC191 SC79

99 Alcanivorax sp. K2-1(AB055204)

Alcanivorax sp. K3-3(AB055205)

$40-5 C 32$

99

${ }_{99} \mathrm{SC46}$

$99-\mathrm{SC151}$

93 uncultured soil bacterium(DQ123671)

sulfur-oxidizing bacterium (AF170424)

$99-\mathrm{SC71}$

Rhodospirillaceae bacterium(DQ401091)

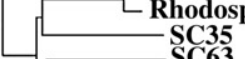

$9 8 \longdiv { 6 3 \square } \quad \mathrm { SC58 }$

52 Caulobacter sp.(AJ227811)

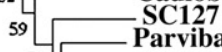

\begin{tabular}{c|c}
70 & SC91 \\
52 & 99 \\
\hline
\end{tabular}

Roseobacter sp. 812(DQ120726)

$99-$ SC179

99 iodide-oxidizing bacterium SE-1(AB15920)

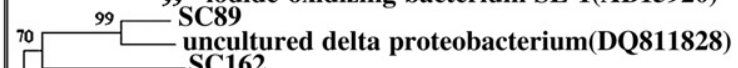

99 SC169

$99-\mathrm{SC182}$

- uncultured Desulfuromonas sp.(EF205264)

$99-$ Malonomonas rubra(Y17712)

99 LC189

85 dissimilatory selenate-respiring bacterium(DQ991964)

$99-\mathrm{SC130}$

Sporosarcina sp. S11-2(DQ514313)

$99+\mathrm{SC} 172$

unidentified Clostridiaceae(U85415)

80

99 .

M086241) Geothrix fermentans(FGU41563)
76 SC170

99 Holophaga sp. oral clone CA002(AF385537)

$99-$ SC137

96 (99 2 SC2 benzene mineralizing consortium clone(AF029039)

99 Flavobacterium psychrophilum(AB219518)

uncultured Flavobacterium sp.(AB219500)

$99\left[\begin{array}{c}\text { uncultured Flavobacterium sp. } \\ \text { SC78 } \\ 99\end{array}\right.$

$99 \quad 99-\begin{gathered}\text { Gillisia } \\ \text { SC147 }\end{gathered}$

$99^{3}-\mathrm{SC73}$

99 Salegentibacter sp.(AY576719)

- Flavobacteriaceae bacterium(DQ640642)

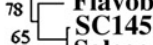

${ }_{65}{ }_{99}$ Salegentibacter flavus(AY682200)

$99-\mathrm{SC139}$ SC139

Chloroflexus aggregans(D32255)

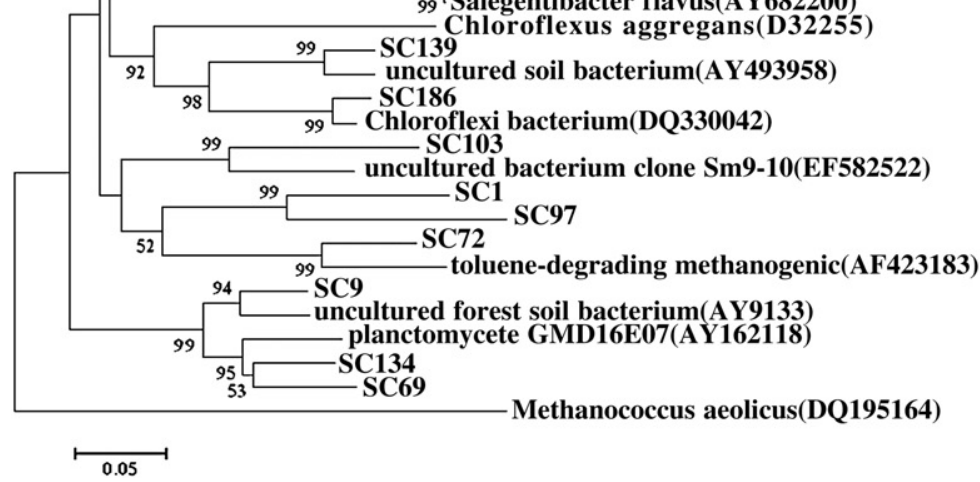

Bacteroidetes

Planctomycetes
Chloroflexi

Unknown

Alphaproteobacteria

Deltaproteobacteria

Fimicutes

Acidobacteria

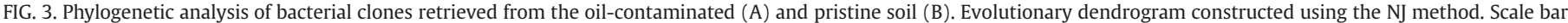
denotes 0.05 indicated changes per nucleotide. (SC, bacterial clone obtained from the contaminated soil; SN, bacterial clone obtained from the pristine soil). 
B

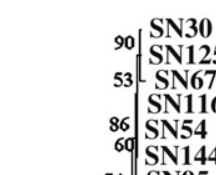

SN144

74 SN95

SN4

$10 \propto\left[\begin{array}{l}\text { SN124 } \\ \text { Pseudo }\end{array}\right.$

Pseudomonas halophila(AB021383)

85 [ SN66

-Salicola marensis(DQ087261)

SN40

100 Marinobacter sp.(DQ412064)

SN159

99 Alcanivorax sp. K3-3(AB055205)

${ }_{89}{ }_{1}$ SN122

100 SN161

- SN149

100 Halomonas salina(AY553063)

100 SN133

$00-$ SN113

Ectothiorhodospiraceae bacterium(EF105754)

uncultured bacterium(AJ966605)

$89-$ SN69

SN166

SN39

${ }_{99} 4 \mathrm{SN}$ SN46

99 SN146

$100-$ uncultured soil bacterium(EF540406)

100 - uncultured Chromatiaceae bacterium(F513942)

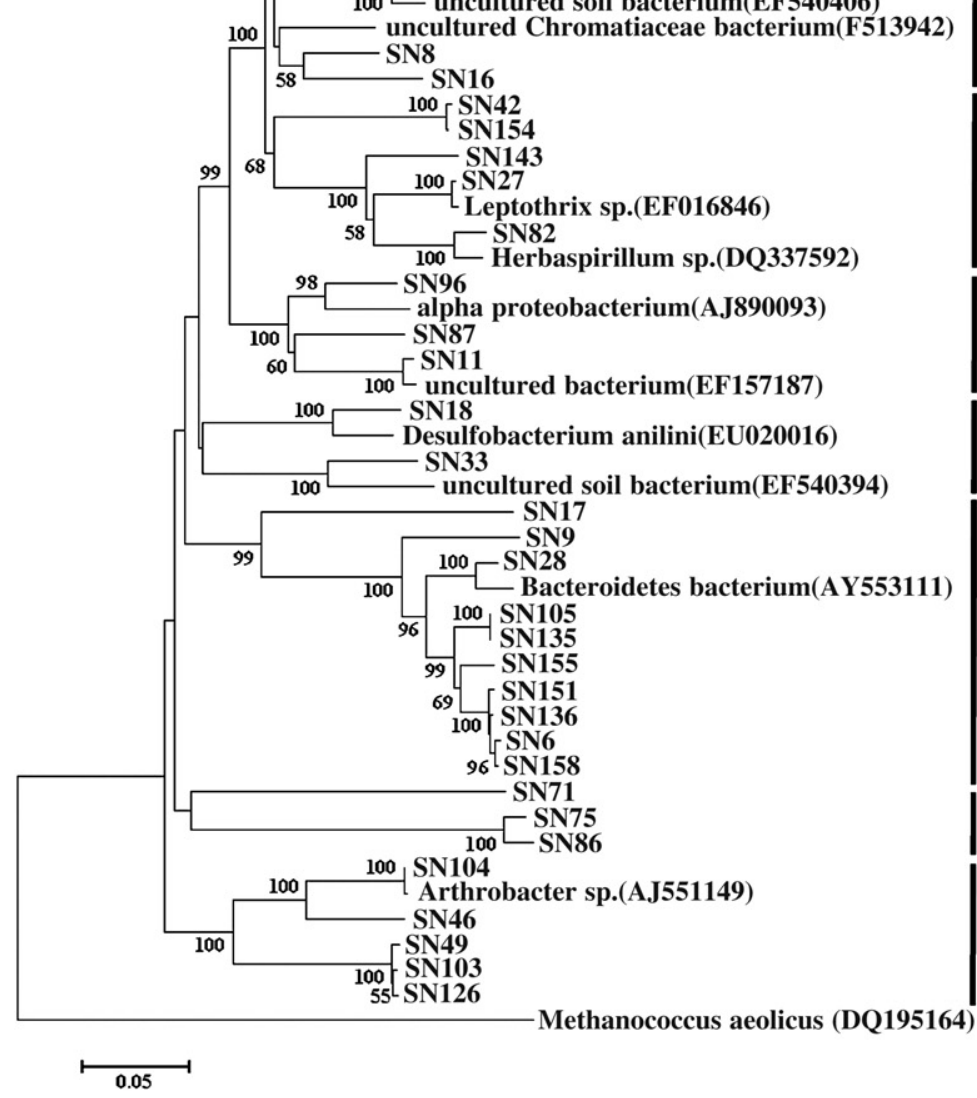

FIG. 3 (continued)
Gammaproteobacteria

Betaproteobacteria

Alphaproteobacteri

Deltaproteobacteria

Bacteroidetes

Unknown

Actinobacteria in marine sediments (26). It has been reported that methanogenic archaea are detected in oil production water and stratal water from oil fields (7), and they are thought to be indigenous microorganisms in oil reservoirs and crude oil (8). Thus, it is likely that methanogens detected here may originate from the contaminating crude oil. Methanogens are strict anaerobes. According to Coates et al., contaminating petroleum can prevent ventilation to soil and stimulate the oxygen consumption, resulting in the formation of anaerobic zones (27). It is possible that anaerobic zones suitable for growth of methanogens are present in the contaminated soil due to the serious oil pollution (Table 1).

The phylogenetic analysis shown in Fig. 2A indicated that 14 OTUs could not be affiliated with known orders of Euryarchaeota, these being grouped into two candidate divisions. Twelve OTUs were 


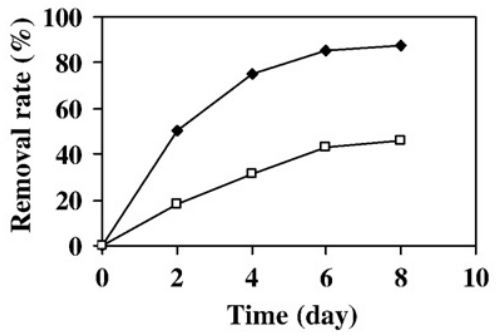

FIG. 4. Degradation of petroleum hydrocarbons in the pristine (open squares) and oilcontaminated (closed rhombuses) soil-slurry systems.

affiliated with candidate division I, which corresponded to rice cluster V. Candidate division I included rather diverse rDNA sequences, suggesting that further studies were needed on this group. The rice clusters have originally been proposed for archaea inhabiting a rice paddy field, while the results of recent studies indicate that members of these groups of archaea are distributed more widely in anoxic environments (25). Rice cluster V-like phylotypes have been detected in oil-contaminated groundwater at the bottom of an underground crude oil storage cavity (25). In this study, one OTU (SCA70) in candidate division I was related to clone KuA18 retrieved from oilcontaminated groundwater with $91 \%$ similarity. Candidate division II included two OTUs that showed high sequence homology with an uncultured archaeon clone retrieved from sediments, and these sequences were associated with clone ASC8 obtained from oilcontaminated unsaturated soil (24). Isolation of these archaea in pure culture or environmental genomic analyses will help to know the ecological roles of these uncultivated archaeal phylotypes in oilcontaminating environments.

All of the four OTUs grouped into Crenarchaeota were related to unidentified environmental clones. The 16S rRNA gene sequences of Crenarchaeota from the upper layer of soils can be recovered on all continents from almost any terrestrial ecosystem. According to Christa et al., non-thermophilic Crenarchaeota found in soils might be chemolithoautotrophic ammonia oxidizers (7).

DGGE profiles and phylogenetic analysis revealed that bacterial community structures were significantly different in the contaminated and pristine soils. Gammaproteobacteria were predominant in the SC library. The majority of Gammaproteobacteria clones (11 of 15 clones) were clustered within the Alcanivorax-Marinobacter-Halomonas complex, members of which are known to degrade petroleum hydrocarbons in various saline environments, such as marine sediments, saline soils, and oil-contaminated seawater. Among them, five clones (SC32, SC46, SC70, SC79, and SC176) were associated with hydrocarbon-degrading Alcanivorax sp. K2-1 and K3-3; four clones (SC10, SC34, SC43, and SC108) showed high similarity with Marinobacter sp. MARC4V (97-99\%) known to degrade polycyclic aromatic hydrocarbons (PAH), and were moderately related to halophilic hydrocarbon-degrading Marinobacter hydrocarbonoclasticus (95-96\%); clones SC13 and SC152 were related to Halomonas spp. (95-98\%), of which Halomonas shengliensis capable of utilizing crude oil was isolated from an oil-contaminated saline soil from Shengli oilfield, China. In addition, Gammaproteobacteria clone SC151 was closely related to uncultured soil bacterium clone PYR10d11 (97\%) associated with the degradation of pyrene in a bioreactor treating soil contaminated with PAH.

Of the other clones in the SC library, two clones were similar with those obtained from anaerobic environments. One clone (SC2) in the class Bacteroidetes was closely related to clone SB-1 (97\%) derived from a sulfate-reducing consortium responsible for benzene-mineralizing, and the other (SC72) was related to clone Eub-3 from a toluene-degrading methanogenic consortium, suggesting the presence of anaerobic niches in the contaminated soil. Additionally, one clone (SC86) in the class Alphaproteobacteria was closely related to Roseobacter sp. 812 (98\%) associated with PAH-degradation.

In contrast to the Gammaproteobacteria group in the SC library, the main Gammaproteobacteria clones (12 of 28 clones) in the SN library were closely related to extremely halophilic Salicola marensis and Pseudomonas halophila (97-99\%) isolated from high saline environments, e.g. saltern and salt lake. Clones clustered within the Alcanivorax-Marinobacter-Halomonas complex were also detected, including three clones (SN122, SN159, and SN161) related to hydrocarbon-degrading Alcanivorax sp. K3-3 (92-94\%), one clone (SN 40) closely related to PAH-degrading Marinobacter sp. MARC4S (98\%), and clone SN111 showing high similarity with halophilic oildegrading $H$. shengliensis (97\%).

It has been reported that Pseudomonas spp. in Gammaproteobacteria and high $\mathrm{G}+\mathrm{C}$ gram-positive Rhodococcus spp. in Actinobacteria are commonly detected bacteria in various oil-contaminating environments (28). In the present study, Pseudomonas- or Rhodococcuslike clones were not detected in the oil-contaminated soil, whereas those affiliated with hydrocarbon-degrading Halomonas, Marinobacter, and Alcanivorax were in abundance. This should be related to relatively high salt content in the soil samples (Table 1). Similar results were observed by Kleinsteuber et al. in a study about the population dynamics within a microbial consortium during growth on diesel fuel in saline environments (29), suggesting that Halomonas, Marinobacter, and Alcanivorax might be the primary hydrocarbondegraders under saline conditions.

In order to compare the potential for hydrocarbon degradation in the contaminated and pristine soil samples, soil-slurry experiments were performed. Both the contaminated and pristine soil-slurry system could biodegrade petroleum hydrocarbons. However, the contaminated soil-slurry system showed the higher TPH degradation rate and 8-day removal, suggesting that the microbial community with substantial hydrocarbon degradation potential had been wellestablished in the contaminated soil. Comparatively abundant hydrocarbon-degrading bacteria-related clones detected in the bacterial SC library may support this conclusion to some extent.

In conclusion, our molecular approach demonstrated that archaeal and bacterial community structures differed significantly between pristine and oil-contaminated soils. The contaminated soil contained more diverse archaeal populations, and included more clones related to halophilic hydrocarbon-degrading microorganisms than those from the pristine soil. These results provide further insights into the composition of microbial communities in heavily oil-contaminated soils.

\section{ACKNOWLEDGMENT}

This work was supported by the National Natural Science Foundation of China (Contract Nos. 50525824, 50621804).

\section{References}

1. Keith, L. H. and Telliard, W. A.: Priority pollutants I-a perspective view, Environ. Sci. Technol., 13, 416-423 (1979).

2. Kasai, Y., Kishira, H., Sasaki, T., Syutsubo, K., Watanabe, K., and Harayama, S.: Predominant growth of Alcanivorax strains in oil-contaminated and nutrientsupplemented sea water, Environ. Microbiol., 4, 141-147 (2002).

3. Hanson, K. G., Nigam, A., Kapadia, M., and Desai, A. J.: Bioremediation of crude oil contamination with Acinetobacter sp. A3, Curr. Microbiol., 35, 191-193 (1997).

4. Popp, N., Schlomann, M., and Mau, M.: Bacterial diversity in the active stage of a bioremediation system for mineral oil hydrocarbon-contaminated soils, Microbiology, 152, 3291-3304 (2006).

5. Labb'e, D., Margesin, R., Schinner, F., Whyte, L. G., and Greer, C. W.: Comparative phylogenetic analysis of microbial communities in pristine and hydrocarboncontaminated Alpine soils, FEMS Microbiol. Ecol., 59, 466-475 (2007).

6. Schleper, C., Jurgens, G., and Jonuscheit, M.: Genomic studies of uncultivated archaea, Nat. Rev. Microbiol., 3, 479-488 (2005). 
7. Magot, M., Ollivier, B., and Patel, B. K. C.: Microbiology of petroleum reservoirs, Antonie Leeuwenhoek, 77, 103-116 (2000).

8. Yoshida, N., Yagi, K., Sato, D., Watanabe, N., Kuroishi, T., Nishimoto, K., Yanagida, A., Kasuragi, T., Kanagawa, T., Kurane, R., and Tani, Y.: Bacterial communities in petroleum oil in stockpiles, J. Biosci. Bioeng., 99, 143-149 (2005).

9. Dojka, M. A., Hugenholtz, P., Haack, S. K., and Pace, N. R.: Microbial diversity in a hydrocarbon- and chlorinated-solvent-contaminated aquifer undergoing intrinsic bioremediation, Appl. Environ. Microbiol., 64, 3869-3877 (1998).

10. Chang, W., Um, Y., and Holoman, T. R. H.: Polycyclic aromatic hydrocarbon (PAH) degradation coupled to methanogenesis, Biotechnol. Lett., 28, 425-430 (2006).

11. Anderson, R. T. and Lovley, D. R.: Hexadecane decay by methanogenesis, Nature 404, 722-723 (2000).

12. Lipson, D. A. and Schmidt, S. K.: Seasonal changes in an alpine soil bacterial community in the Colorado Rocky Mountains, Appl. Environ. Microbiol., 70, 2867-2879 (2004).

13. Webster, G., Parkes, R. J., Fry, J. C., and Weightman, A. J.: Widespread occurrence of a novel division of bacteria identified by $16 \mathrm{~S}$ rRNA gene sequences originally found in deep marine sediments, Appl. Environ. Microbiol., 70, 5708-5713 (2004).

14. National Environmental Protection Bureau: Monitoring and determination methods for water and wastewater, p. 491-495. China Environmental Science Press, Beijing (2002) (in Chinese).

15. Wang, Z., Fingas, M., and Li, K.: Fractionation of a light crude oil and identification and quantification of aliphatic, aromatic and biomarker compounds by GC-FID and GC-MS, Part I, J. Chromatogr. Sci., 32, 361-366 (1994).

16. Ryden, J. C. and Syers, J. K.: Origin of the labile phosphate pool in soils, Soil Sci., 123, 353-361 (1977).

17. Tsai, Y. L. and Olson, B. H.: Rapid method for direct extraction of DNA from soil and sediments, Appl. Environ. Microbiol., 57, 1070-1074 (1991).

18. Muyzer, G., de Waal, E. C., and Uitterlinden, A. G.: Profiling of complex microbial populations by denaturing gradient gel electrophoresis analysis of polymerase chain reaction-amplified genes coding for $16 \mathrm{~S}$ rRNA, Appl. Environ. Microbiol., 59, 695-700 (1993).

19. Baker, G. C., Smith, J. J., and Cowan, D. A.: Review and reanalysis of domainspecific $16 \mathrm{~S}$ primers, J. Microbiol. Methods, 55, 541-555 (2003).
20. Amann, R. I., Ludwig, W., and Schleifer, K. H.: Phylogenetic identification and in situ detection of individual microbial cells without cultivation, Microbiol. Rev., 59, 143-169 (1995).

21. Brofft, J. E., McArthur, J. V., and Shimkets, L. J.: Recovery of novel bacterial diversity from a forested wetland impacted by reject coal, Environ. Microbiol., 4, 764-769 (2002).

22. Ce'bron, A., Coci, M., Garnier, J., and Laanbroek, H. J.: Denaturing gradient gel electrophoretic analysis of ammonia-oxidizing bacterial community structure in the Lower Seine River: impact of Paris wastewater effluents, Appl. Environ. Microbiol., 70, 6726-6737 (2004).

23. Valenzuela-Encinas, C., Neria-González, I., Alcántara-Hernández, R. J., EnríquezAragón, J. A., Estrada-Alvarado, I., Hernández-Rodríguez, C., Dendooven, L., and Marsch, R.: Phylogenetic analysis of the archaeal community in an alkaline-saline soil of the former Lake Texcoco (Mexico), Extremophiles, 12, 247-254 (2008).

24. Kasai, Y., Takahata, Y., Hoaki, T., and Watanabe, K.: Physiological and molecular characterization of a microbial community established in unsaturated, petroleumcontaminated soil, Environ. Microbiol., 7, 806-818 (2005).

25. Watanabe, K., Kodama, Y., Hamamura, N., and Kaku, N.: Diversity, abundance and activity of archaeal populations in oil-contaminated groundwater accumulated at the bottom of an underground crude oil storage cavity, Appl. Environ. Microbiol., 68, 3899-3907 (2002).

26. Zinder, S. H.: Physiological ecology of methanogens, in: J. G. Ferry (Ed.), Methanogenesis: Ecology, Physiology, Biochemistry and Genetics, Chapman \& Hall, New York, 1993, pp. 128-206 (1993).

27. Coates, J. D., Chakraborty, R., Lack, J. G., O’Connor, S. M., Cole, K. A., Bender, K.S. and Achenbach, L. A.: Anaerobic benzene oxidation coupled to nitrate reduction in pure culture by two strains of Dechloromonas, Nature, 411, 1039-1043 (2001).

28. Hamamura, N., Olson, S. H., Ward, D. M., and Inskeep, W. P.: Microbial population dynamics associated with crude-oil biodegradation in diverse soils, Appl. Environ. Microbiol., 72, 6316-6324 (2006).

29. Kleinsteuber, S., Riis, V., Fetzer, I., Harms, H., and Müller, S.: Population dynamics within a microbial consortium during growth on diesel fuel in saline environments, Appl. Environ. Microbiol., 72, 3531-3542 (2006). 